Proc. SPIE, Unmanned/Unattended Sensors and Sensor Networks X, 9248-33 (nov 2014)

doi: $10.1117 / 12.2074284$

Copyright 2014 Society of Photo-Optical Instrumentation Engineers. One print of electronic copy may be made for personal use only. Systematic reproduction and distribution, duplication of any material in this paper for a fee or for commercial purposes, or modification of the content of the paper are prohibited.

\title{
Encounter Detection to Improve Navigation in a Group of Unattended Vehicles
}

\author{
M.G.A. Ruizenaar (marcel.ruizenaar@tno.nl) \\ TNO Technical Sciences, The Hague, The Netherlands
}

\begin{abstract}
Research was conducted on groups of unattended vehicles performing some collaborative tasks. Navigation of these vehicles and more specifically, knowing each other's position, is mandatory. In densely built environments, forests, etc., GPS is not always available or accurate. Using information about encounters between the vehicles may significantly improve position information. Detecting encounters can be done very easily with low cost sensors. TNO developed and patented an innovative technology that uses information on encounters to improve the position information. The first promising results show that information on encounters can be used in standard integrated INS/GNSS navigation systems to aid the navigation solution. To increase the number of encounters, a group of unattended vehicles may deliberately try to encounter each other if their navigation solution becomes less certain during GNSS outages.
\end{abstract}

Keywords: Encounter, Navigation, Unattended, INS, GNSS, Kalman, Unaided, Proximity

\section{INTRODUCTION}

Nowadays, Global Navigation Satellite Systems (GNSS) are the main type of navigation system in most applications. There are several satellite navigation systems like the European Galileo or the Chinese Beidou, but the most widely used is still the US Global Positioning System (GPS). The wide use of these systems has led to the development of low-cost localization systems. One major problem of these satellite localization systems is that they rely on the reception of very weak radio signals transmitted from satellites orbiting in space. From these signals, range measurements to individual satellites are derived from which the location can be calculated. Obstructions like buildings, trees etc. may further weaken the signals, making individual range measurements impossible. Another problem is the so-called multi-path: a satellite signal is reflected, perhaps even a multiple times, from surfaces. This causes the measured range to be much larger, and adversely affect the localization based on these measurements. Both the weakening of satellite signals and multi-path occurs in for instance dense built urban environments, urban canons, in or near tunnels, under bridges, but also forests etc. In many of such situations, GNSS reception may not be reliable or is not possible at all. Furthermore, even in non-military situations, GNSS signals may be jammed ${ }^{2}$ or spoofed, both intentionally or unintentionally. Because of the very weak satellite signals, small battery powered jammers are very easy to build. Spoofing a GPS signal, deliberately making the receiver report false locations, has already been demonstrated ${ }^{I}$.

Because of these problems, there is a need for alternative ways of localization in GNSS degraded and/or denied environments. Much research effort is done on using for instance cameras or radio frequency beacons. Cameras can be used to observe a change in pose with respect to the surroundings. Radio frequency beacons may be used in a similar way as satellites. Also much effort is done in research on the use of so-called signals of opportunity. Signals of opportunity are received or sensed signals that are not intended for navigation, but can be used for that purpose ${ }^{3}$. Signals of opportunity may be transmitted radio or television signals, but also signals of Wifi base stations. Also the Earth magnetic field may serve that purpose. Ref $^{3}$ describes the use of this Earth magnetic field for navigation.

This paper describes an innovative alternative concept to aid navigation in degraded GNSS environments. It uses information on encounters between navigating unattended vehicles. Most unattended vehicles are equipped with a safety device: a proximity radar that detects the close proximity of other objects. If another object is too close, the unattended 
vehicles will stop or perform some intelligent action to avoid a collision. The same proximity radar may be altered to detect other unattended vehicles and exchange information with them. In that case, it may detect encounters between other unattended vehicles, and generate useful aiding information to the navigation system.

The questions, addressed in this paper, are: if an encounter is detected between two navigating vehicles, how can this be translated to aiding information and how can it be used to aid the navigation system?

\section{LOCALIZATION WITH ENCOUNTER DETECTION}

In 2008, TNO has developed and patented ${ }^{5}$ a technology that uses information about encounters between navigating objects to generate location information. It was discovered that if two objects (two cars for instance) detect an encounter between each other, new information was generated that was not known before. This is illustrated in Figure 1. Both cars are equipped with a proximity detection device, and an encounter will be detected if the relative range between the cars is below some value $\mathrm{L}_{\mathrm{d}}$. In general this detection range is not constant and depends on the proximity detection method and probably on the environment. A laser based proximity detection device for instance, may have a very well defined detection range. A radio signal strength based device on the other hand, may have an expected detection range that highly depends on the reflecting properties of surrounding objects. The expected detection range $\mathrm{L}_{\mathrm{d}}$ defines a sphere around each vehicle. Once detected, the other car is therefore "expected" to be within that sphere. At first detection, it is expected to be on the boundary of the sphere. It may seem obvious, but if two objects are known to be in close proximity (=detection), their locations must approximately be the same (=information).

$$
\bar{p}_{A \mid \text { det }} \cong \bar{p}_{B \mid \text { det }}
$$

This fact or piece of information was not known before the encounter detection. The detection therefore generates new navigation related information.

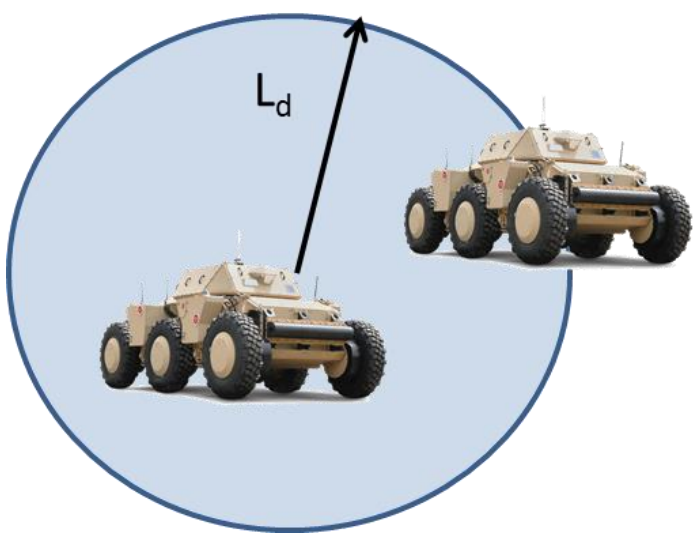

Figure 1. Two objects (e.g. cars) come in close proximity of each other. An encounter is detected if their relative range is less than some expected detection range $\mathrm{L}_{\mathrm{d}}$.

The question is: what can we do with this piece of information? A single piece of information may not be very informative. But many encounters between a multiple of navigating objects, possibly combined with other pieces of information about for instance expected speed, roads etc. may lead to improved location information.

Initially, we processed the encounter information with a so-called Bayesian Belief Network (BBN) ${ }^{6}$, based on Bayes probabilistic rule ${ }^{8}$. This BBN was combined with map information about roads and assumptions on travelling speed of the vehicles. No further navigation related information was input to the network. Several simulations were done, and some practical experiments ${ }^{7}$. The results clearly showed that it was possible to recover position information, solely from the encounter information, map information and some simple assumptions on travelling speed. However, for several reasons, this BBN based method was not very practical.

1) Information was processed by a central computer, which is not preferred in the case of unattended vehicles. Further, maps of roads and preplanned routes are not always available and the speed of the vehicle is not always known. 
2) A lot of encounters are needed. In contrast, the probability of two unattended vehicles encountering each other in a large area is very low. Increasing the detection range increases the availability of encounter information, but decreases the quality of the information. A strategy to overcome this might be to have the unattended vehicles deliberately encounter each other.

3) The BBN grows exponentially with the number of encounters and the number of vehicles. This makes it computationally less attractive.

The next section discusses how encounter information can be used in a more practical way in standard integrated navigation systems.

\section{ENCOUNTER INFORMATION IN INTEGRATED NAVIGATION SYSTEMS}

Inertial Navigation Systems (INS) use the information of inertial sensors to calculate position, velocity and attitude. Inertial sensors, also named Inertial Measurement Units (IMU), are a combination of accelerometers that measure acceleration and gyroscopes that measure angular rate. Inertial sensors measure changes with respect to an inertial reference frame. In that sense they do not rely on the existence of any human built infrastructure (like GPS). This is one of the main advantages of an INS: it cannot be disturbed by any signal from outside the navigation system. One of the major disadvantageous of an INS is its well-known drift, caused by small inertial sensor errors that cumulate in time.

This drift makes the INS navigation solution $\left[\begin{array}{ccc}\overline{\tilde{p}} & \overline{\tilde{v}} & \overline{\tilde{\theta}}\end{array}\right]^{T}$ (also called the INS state) become more uncertain in time. In this state vector, $\overline{\tilde{p}}$ is the position, $\overline{\tilde{v}}$ is the velocity and $\overline{\tilde{\theta}}$ the attitude, all vectors. A tilde is used to indicate that the INS state is an estimate of the true navigation state $\left[\begin{array}{lll}\bar{p} & \bar{v} & \bar{\theta}\end{array}\right]^{T}$. The superscript ${ }^{\mathrm{T}}$ is used to denote a vector or matrix transposition. Depending on the quality of the inertial sensors used, the uncertainty may grow exponentially in time. If low-cost inertial sensors are used, unaided inertial navigation is usually limited to in the order of half a minute. For this reason, practical INS-based navigation systems are always used in combination with other sensors. Such a combined system is usually called an integrated navigation system. It is widely known and described in for instance ${ }^{9}$. The remainder of this paper focusses on the way to seamlessly combine encounter information in such integrated navigation systems. Figure 2 shows a typical lay-out of an integrated navigation system in which an INS is integrated with information from other aiding sensors.

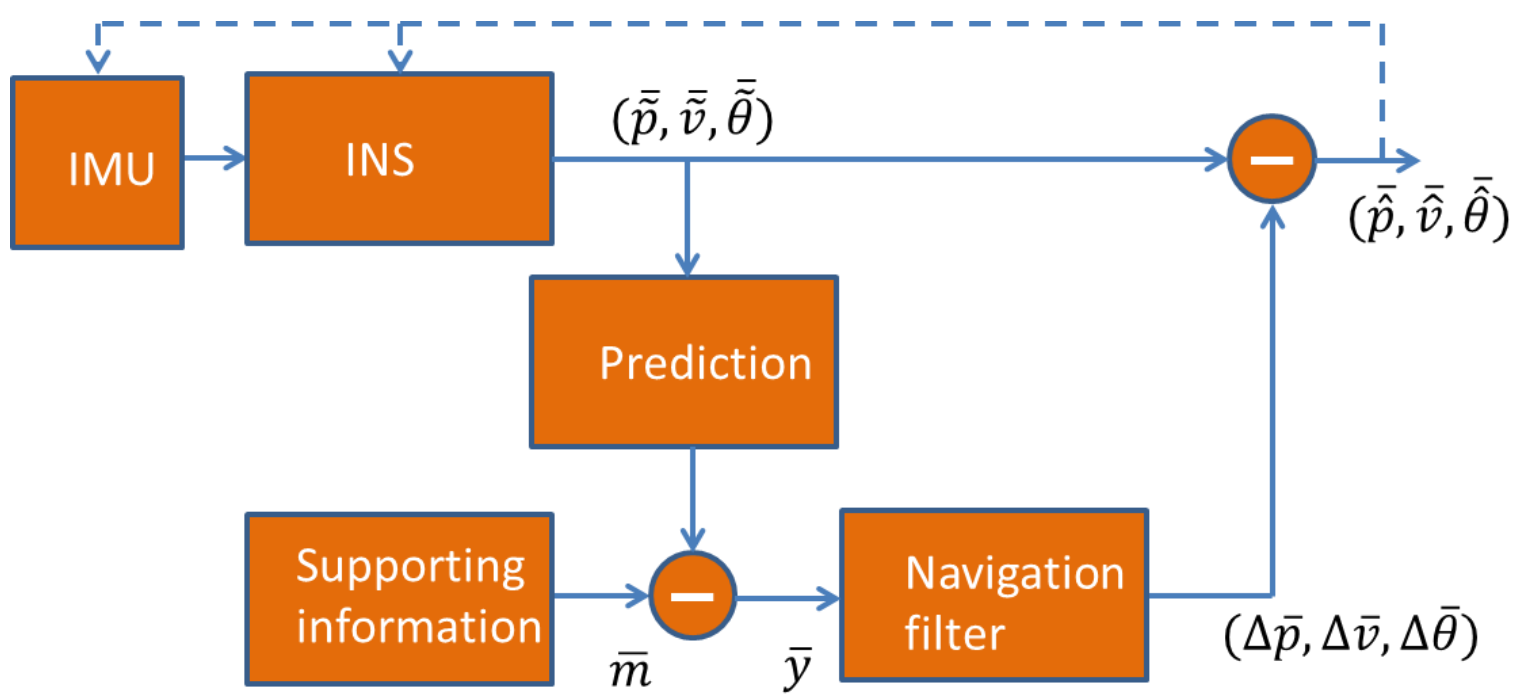

Figure 2. Typical lay-out of an INS based navigation system that is supported by other sensors. A navigation filter (usually a Kalman filter) estimates the INS state errors and corrects the INS state for it.

Suppose at some time $\mathrm{t}$ supporting information $\bar{m}$ is available. $\bar{m}$ can be a single measurement of a scalar quantity (like for instance a height measurement from a barometer or a single range measurement to a beacon), a vector (like for instance a position measurement from a GNSS receiver), but it can also be a combination of scalars and vectors. The multi-dimensional value of the measurement vector is predicted based on the INS state $\left[\begin{array}{lll}\overline{\tilde{p}} & \overline{\tilde{v}} & \overline{\tilde{\theta}}\end{array}\right]^{T}$ and possibly other 
a-priori known information. The INS state deviates from the true navigation state $\left[\begin{array}{lllll}\bar{p} & \bar{v} & \bar{\theta}\end{array}\right]^{T}$ by an error $\left[\begin{array}{lll}\Delta \bar{p} & \Delta \bar{v} & \Delta \bar{\theta}\end{array}\right]^{T}$. The difference between the predicted measurement and the actual measurement is calculated, resulting in a measurement difference $\bar{y}$. A navigation filter, usually a Kalman filter, is used to estimate the INS state error from these measurement differences. The error estimate is subtracted from the erroneous INS state, resulting in a best estimate $\left[\begin{array}{lll}\overline{\hat{p}} & \overline{\hat{v}} & \overline{\hat{\theta}}\end{array}\right]^{T}$ of the true state. A hat is used to indicate the best estimate. For practical reasons, the best estimate is regularly fed back to the INS. This feedback is indicated by the dashed line. A well-known type of integrated navigation system is the INS/GNSS integrated navigation system, of which different representations are available ${ }^{9}$. Nowadays, complete low-cost solutions are available that combine GPS and an INS in an integrated manner in a single chip ${ }^{10}$.

The Kalman filter in such an INS based integrated navigation system uses a set of recursion formulas:

$$
\begin{gathered}
P_{k+1}^{-}=\Phi P_{k} \Phi^{T}+Q \\
K=\frac{P_{k+1}^{-} H^{T}}{H \cdot P_{k+1}^{-} H^{T}+R} \\
P_{k+1}=(I-K \cdot H) P_{k+1}^{-} \\
\bar{x}_{k+1}=\Phi \cdot \bar{x}_{k}+K\left(\bar{y}_{k+1}-H \cdot \Phi \cdot \bar{x}_{k}\right)
\end{gathered}
$$

In which, $P_{k}$ is the error covariance matrix at sample time $\mathrm{t}_{\mathrm{k}}$ and $P_{k+1}^{-}$is the estimate of the error covariance of the next sample time before measurements are taken into account. $\Phi$ is the transition matrix. Q is the noise covariance matrix. $\mathrm{K}$ is the Kalman gain matrix. $\mathrm{H}$ is the measurement matrix. $\mathrm{R}$ is the measurement noise matrix. $\bar{x}_{k}$ is the INS error state vector to be estimated: $\bar{x}_{k}=\left[\begin{array}{lll}\Delta \bar{p} & \Delta \bar{v} & \Delta \bar{\theta}\end{array}\right]^{T}$.

The basics of Kalman filtering are well described in the literature ${ }^{9},{ }^{11},{ }^{12}$. For the sequel, only two matrices are important: the measurement matrix $\mathrm{H}$, relating the aiding measurements to the error states: $\bar{y}_{k}=H \cdot \bar{x}_{k}$, and the measurement noise matrix R, representing the amount of noise on the measurements. For each source of information, a measurement matrix must be found that satisfies this equation. In some cases, finding this measurement matrix $\mathrm{H}$ is trivial. An example is the measurement equation for a GNSS position measurement.

$$
\bar{y}=\left[\begin{array}{lll}
I & O & O
\end{array}\right] \cdot\left[\begin{array}{lll}
\Delta \bar{p} & \Delta \bar{v} & \Delta \bar{\theta}
\end{array}\right]^{T}
$$

With I a $3 \times 3$ identity matrix and $\mathrm{O}$ a $3 \times 3$ zeros matrix. This results in a measurement matrix $H=\left[\begin{array}{lll}I & O & O\end{array}\right]$.

The measurement noise matrix $\mathrm{R}$ in this example is a diagonal matrix with the $\mathrm{x}, \mathrm{y}$ and $\mathrm{z}$ measurement variances on the diagonal elements.

Now the question is: if we have detected an encounter between two navigating vehicles, how can we translate this information to useful measurement matrices $\mathrm{H}$ and $\mathrm{R}$ for both corresponding navigation systems? If an encounter takes place, both navigation systems can communicate with each other and mutually exchange information on each other's position estimates and certainty of these position estimates. As a first thought experiment, we have taken the position estimate of vehicle $\mathrm{B}$ as a direct reference measurement for the navigation system of vehicle $\mathrm{A}: \bar{y}=\overline{\tilde{p}}_{A}-\overline{\hat{p}}_{B}=\Delta \bar{p}$, with $\overline{\hat{p}}_{B}$ the current best position estimate of vehicle B. The measurement matrix is then: $H=\left[\begin{array}{lll}I & O & O\end{array}\right]$. For the variance of this measurement (matrix R) we have taken the sum of the variance of vehicle B's position estimate and the variance of the encounter detection. As the other vehicle can be within the sphere with radius $\mathrm{L}_{\mathrm{d}}$ of the first vehicle, the measurement noise matrix becomes:

$$
R=\operatorname{diag}\left(P_{k, B}\left(\Delta \bar{p}_{B}\right)\right)+I \cdot L_{d}
$$

Off course, the same applies for the measurement, measurement matrix and measurement noise matrix for vehicle B.

\section{SIMULATION}

Some simulations have been done to investigate the performance improvements that can be achieved using encounter information. The trajectories of two vehicles (A and B) were simulated. These trajectories were created by using a few waypoints for each trajectory and fitting a polynomial on these waypoints. These polynomials were sampled with a frequency of $100 \mathrm{~Hz}$, and, using backward differencing, simulated accelerometer and gyroscopes signals were derived. Subsequently integrating these accelerometer and gyroscope signals resulted in a trajectory that was taken as a baseline 
"true" trajectory. These baseline trajectories for both vehicles are plotted in Figure 3 below. A simulation model of a Loosely coupled INS/GNSS integrated navigation system was built in Matlab and was expanded to incorporate the encounter information. This type of system uses position information from a GNSS receiver to aid the INS.

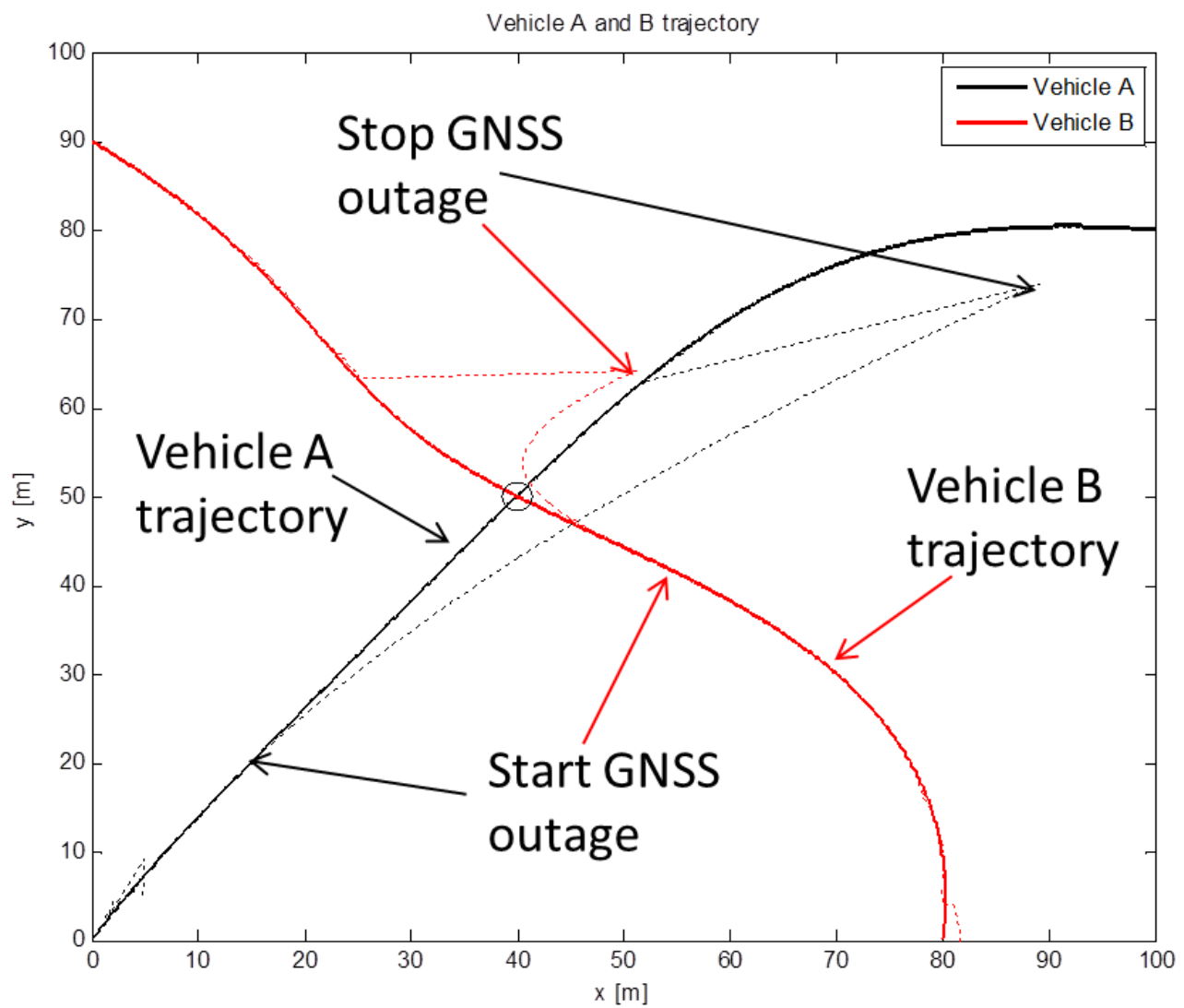

Figure 3. Plot of baseline "true" trajectories for vehicle A and B. The dotted lines represent trajectories where GNSS aiding was lost during some period of time. The INS then start to drift. At some time, GNSS aiding commences again and the position drift is quickly corrected.

Noise sources of $400 \mu \mathrm{g} / \sqrt{ } \mathrm{Hz}$ for the accelerometers and $0.005 \mathrm{deg} / \sqrt{ } \mathrm{Hz}$ for the gyroscopes, which are typical for lowcost MEMS inertial sensors, were added to the simulated sensor outputs. For the sensor biases we have taken random values from a normal distribution with standard deviation of $1 \mathrm{mg}$ for the accelerometers and $50 \mathrm{deg} / \mathrm{hr}$ for the gyroscopes. These values remained fixed during the simulation of a trajectory. Other inertial sensor errors were not taken into account. The simulated GNSS position measurements were taken to be the true (baseline) position with normal distributed noise of $1 \mathrm{~m}$ standard deviation added to it. The Kalman filter estimated the INS position errors, velocity errors and attitude errors. No other states were estimated.

During some part of the trajectory we omitted the GNSS position updates, effectively simulating a GNSS outage. This resulted in the INS/GNSS solution drifting away. This "drifting" position is also plotted in Figure 3 with a dotted line. Once GNSS position updates were available again, the position estimate quickly converged back to nearly the baseline solution (the sharp turns in the dotted lines).

Figure 4 shows the results for a specific example. The figure shows the INS/GNSS position error in $\mathrm{x}$ and $\mathrm{y}$ direction of both vehicles as a function of time. The $\mathrm{z}$ direction has been omitted. Usually this direction is not interesting if we assume navigation takes place in the horizontal plane. Further vertical position errors can easily be estimated using barometer data. The solid lines show the corrected position errors when encounter information is taken into account. The dashed lines show the position errors if encounter information is not taken into account. The lower plots in Figure 4 
show the square root of the variance of the $\mathrm{x}$ and $\mathrm{y}$ position error states estimated by the Kalman filter as indicated by the $P_{k}$ matrix. These values indicate how certain the Kalman filter is of its estimates. The encounter is detected at approximately 45 seconds. As can be seen, at the time of the encounter, the Kalman filter of vehicle B is much more certain about its position estimates then that of vehicle A. Therefore vehicle B is acting as a beacon to vehicle A. The position drift of vehicle $\mathrm{A}$ is corrected a lot and the uncertainty decreases. As vehicle B is already very certain, its position and uncertainty are not updated noticeably.
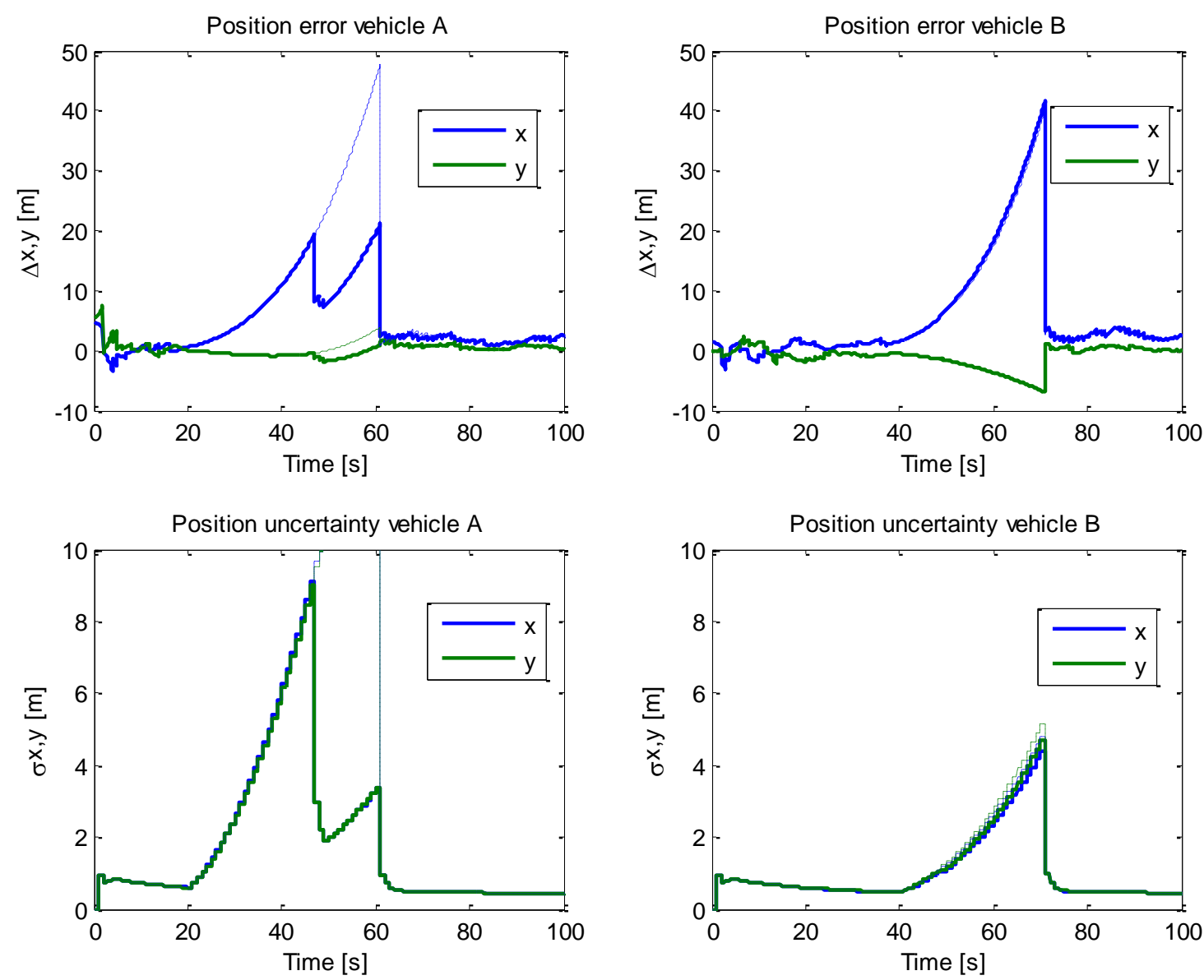

Figure 4. Example results of a INS/GNSS system, aided by encounter information. At some time, GNSS information stops, causing the position errors to increase. Encounter information at approximately 45 seconds, reduced the drift.

Figure 5 presents the results of another example. In this case, the uncertainty of vehicle B is higher. It acts as a more "vague" beacon to vehicle A. In this specific case, vehicle A is still corrected by the information. However, although the uncertainty of vehicle B has decreased, the error of vehicle B has increased a little. 

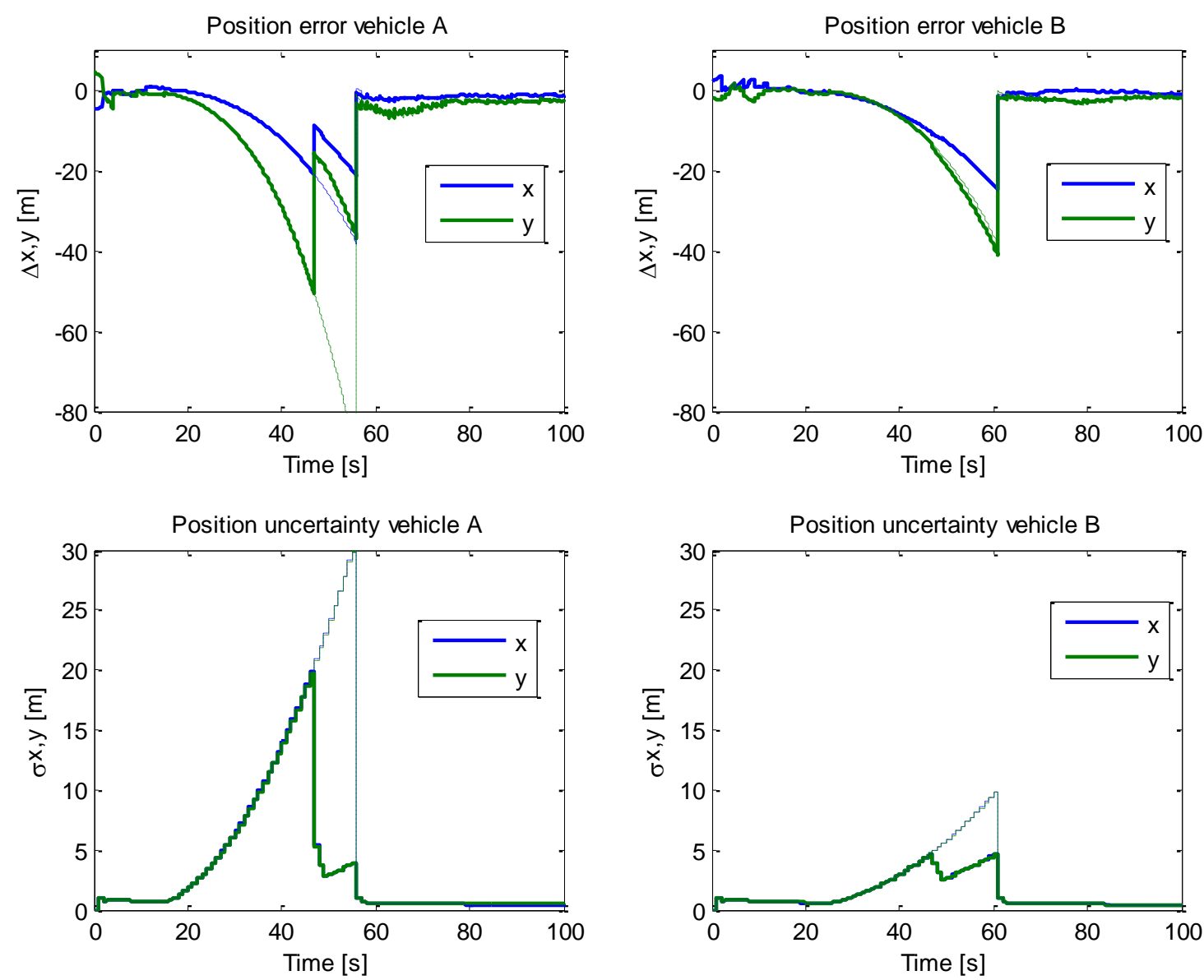

Figure 5. Another example of an INS/GNSS system, aided by encounter information. In this case, vehicle B is less certain about its position. Vehicle A can still benefit from this information, but the position errors of vehicle B increases slightly.

\section{DISCUSSION}

The first preliminary results show that encounter information can be used to aid a standard INS/GNSS integrated navigation system in the event of GNSS outages. In the examples, two vehicles are used with a single encounter. Obviously, results improve if there are more encounters, generating more information. This can be achieved by increasing the number of vehicles. As each encounter is only processed by the two vehicles involved in the encounter, the method can easily be expended to more vehicles. It is however also possible that unattended vehicles deliberately try to encounter each other when they become less certain about their position. Effectively this becomes a way of cooperative navigation.

The results also show that the position estimates itself might become worse, depending on the certainty of the other vehicle. The estimation of the certainty always becomes better. This aspect needs to be investigated further. 


\section{REFERENCES}

[1] Shepard, D. et. al., "Drone Hack", GPS World, August 2012, pp. 30-33.

[2] Grabowski, J., "Personal Privacy Jammers", GPS World, April 2012, pp. 28-37.

[3] John F. Raquet, "Using Pseudolites, Beacons, and Signals of Opportunity", NATO panel STO-EN-SET-197, oct. 2013, Brussels, Belgium.

[4] John F. Raquet, Jeremiah A. Shockley, and Kenneth A. Fisher, "Determining Absolute Position Using 3-Axis Magnetometers and the Need for Self-Building World Models", NATO panel STO-EN-SET-197, oct. 2013, Brussels, Belgium.

[5] M.G.A. Ruizenaar, R.M. Neef, S.A. van Merriënboer, "Location information based upon electronic tags", Patent application WO2009NL50679.

[6] Judea Pearl, "Probabilistic Reasoning in intelligent systems", ISBN 1-55860-479-0

[7] Marcel Ruizenaar, "Smart Tags, een sociaal netwerk concept voor het in kaart brengen van bezoekersstromen (Smart Tags, a social network concept to map the flow of visitors)", TNO report, TNO-DV 2009 B542.

[8] http://en.wikipedia.org/wiki/Bayes'_theorem

[9] P.D. Groves (2008). "Principles of GNSS, inertial, and multi-sensor integrated navigation systems", ISBN-13: 978-1-58053-255-6

[10] http://www.prnewswire.com/news-releases/vectornav-releases-the-worlds-first-gps-aided-inertial-navigationsystem-on-a-chip-163725606.html

[11]R. E. Kalman. "A new approach to linear filtering and prediction problems", Transaction of the ASME-Journal of Basic Engineering, pages 35-45, March 1960.

[12] G.Welch and G. Bishop. "An introduction to the kalman filter", Department of Computer Science, University of North Carolina at Chapel Hill, 2006. 